\title{
X-Ray Supernovae
}

\author{
Stefan Immler ${ }^{1}$ and Walter H.G. Lewin ${ }^{2}$ \\ 1 Astronomy Department, University of Massachusetts, Amherst, MA 01003 \\ 2 Center for Space Research and Department of Physics, \\ Massachusetts Institute of Technology, Cambridge, MA 02139-4307
}

\begin{abstract}
We present a review of X-ray observations of supernovae (SNe). By observing the $(\sim 0.1-100 \mathrm{keV})$ X-ray emission from young SNe, physical key parameters such as the circumstellar matter (CSM) density, mass-loss rate of the progenitor and temperature of the outgoing and reverse shock can be derived as a function of time. Despite intensive search over the last $\sim 25$ years, only 15 SNe have been detected in $\mathrm{X}$-rays. We review the individual X-ray observations of these $\mathrm{SNe}$ and discuss their implications as to our understanding of the physical processes giving rise to the $\mathrm{X}$-ray emission.
\end{abstract}

\section{Introduction}

To date, several thousand supernovae ( $\mathrm{SNe}$ ) have been discovered in the optical, whereas only $\sim 30 \mathrm{SNe}$ have been detected in the radio and 15 in the X-ray band $(\sim 0.1-100 \mathrm{keV})$. Neutrinos have been recorded from one nearby supernova only. The reasons for this large diversity lies in the simple facts that (i) the flux levels of the various constituents, as well as the sensitivity of the available detectors/telescopes are vastly different, and (ii) systematic or automatic searches for SNe are only made in the optical. Prior to the beginning of automatic searches in the late 1980s 14], about two dozen SNe were reported per year in the optical. This number has now grown to about several hundred per year (e.g. 248 confirmed detections in the year 2001]). The detection of two dozen neutrinos from SN 1987A [4] 32] was only possible because of its close proximity at a distance of about $50 \mathrm{kpc}$ in the Large Magellanic Cloud. SNe have been detected up to a redshift of $z \sim 1.7$ (SN 1997ff [56]) in the optical and IR, and up to $z \sim 0.022$ (SN 1988Z; $\sim 100 \mathrm{Mpc}$ ) in both the radio 72 and X-rays 17].

Based on the presence or absence of hydrogen lines in their spectra, SNe are classified as Type II and I, respectively (see the Chap. by Turatto and references therein). No Type Ia SN has been detected to date in either the radio or in X-rays. These SN are believed to be nuclear detonations of carbon+oxygen $(\mathrm{C}+\mathrm{O})$ white dwarfs when they exceed the Chandrasekhar limit through accretion (48,81 and references therein). Due to recurrent pulsations of the progenitor, fast shells (several $\times 100 \mathrm{~km} \mathrm{~s}^{-1}$ ) are ejected from the progenitor. During periods of quiescence, no stellar wind is blown into the CSM due to the low mass of the progenitor. This leads to the formation of a complicated, yet low density CSM structure with interacting shells of different expansion speeds. The

\footnotetext{
${ }^{1}$ from the Asiago Supernova Catalogue: http://merlino.pd.astro.it/ supern
} 
relatively slow expanding ejecta $\left(\lesssim 5,000 \mathrm{~km} \mathrm{~s}^{-1}\right)$ can hence not form a shock region that could give rise to thermal X-ray or radio emission (see below). Since no X-ray emission has been recorded from a Type Ia SN to date, and is neither expected at a detectable flux level, we will not discussed Type Ia's here further.

This leaves us still with a zoo of different types: Ib, Ic, IIP, IIL, IIb, and IIn all of which are believed to be the result of the core collapse of a massive star ( $\gtrsim 10 M_{\odot}$ ZAMS; e.g. [6.30] and references therein). The dividing line between these types is not always clear, and there are numerous examples whereby an original designation was later changed as the spectra and the light curves evolved in time (SN 1998bw, for example, was classified as both Ib and Ic at different times during its evolution). The more detailed information is available on a particular SN, the more difficult it becomes to 'squeeze' it into a category of known types. This has led to the introduction of the 'peculiar' SNe, abbreviated 'pec', and it is not so surprising that two of the best studied SNe, 1987A and 1993J, have both obtained the 'pec' distinction from several authors (e.g. 20.66]).

Here follows a verbatim quote from Kurt Weiler: "My general rule of thumb is: any well observed $S N$ is peculiar. Only the poorly observed ones fit nice classifications - for classifications, bad data is good. Think of it this way: at a distance, and in the dark, all cats fit a nice category. With close examination, and in the light, they are all individuals."

The X-ray luminosities of all detected SNe are in the range $10^{37}-10^{41} \mathrm{ergs} \mathrm{s}^{-1}$. A compilation of all $15 \mathrm{X}$-ray SNe is given in Tab. 1 P. They dominate the total luminosity of the SNe starting at an age of about one year. The X-ray emission, as well as the radio emission, are largely the result of the interaction of the ejecta with the CSM, which is present as a result of the wind of the SN progenitor 11, 69. Progenitors of Type II SNe following core collapse of massive massive stars have high mass loss rates $\left(\dot{M} \sim 10^{-4}-10^{-6} \mathrm{M}_{\odot} \mathrm{yr}^{-1}\right)$ and low wind velocities of typically $v_{\mathrm{w}} \sim 10 \mathrm{~km} \mathrm{~s}^{-1}$. Type $\mathrm{Ib} / \mathrm{c}$ SNe, likely to originate from more compact stars, have lower mass-loss rates $\left(\dot{M} \sim 10^{-5}-10^{-7} M_{\odot} \mathrm{yr}^{-1}\right)$ and significantly higher wind velocities of $v_{\mathrm{w}} \lesssim 1,000 \mathrm{~km} \mathrm{~s}^{-1}$.

Shocks are formed as the ejecta plow into the CSM: (i) the 'circumstellar' or 'forward shock' (also called 'blastwave'), and (ii) the so-called 'reverse shock'. In the early phase after the $\mathrm{SNe}$, the speed of the ejecta (i.e., of the circumstellar shock) is of the order of $\sim 10^{4} \mathrm{~km} \mathrm{~s}^{-1}$. The speed of the reverse shock is $\sim 10^{3} \mathrm{~km} \mathrm{~s}^{-1}$ lower. Depending on the density profile of the ejecta, as they emerge from the star, the density behind the reverse shock (i.e. at larger radii than that of the reverse shock) can be 5-10 times higher than the density behind the circumstellar shock (i.e. at smaller radii than the circumstellar shock front; cf. Fig. 1 in the Chap. by Chevalier \& Fransson). The temperature behind the circumstellar shock can be as high as $10^{9-10} \mathrm{~K}$, whereas the temperature behind the reverse shock is significantly lower $\left(10^{7-8} \mathrm{~K}\right)$. The soft X-ray $(\lesssim 5 \mathrm{keV})$ emission is therefore generally explained in terms of thermal radiation from the reverse shock, whereas the higher energy X-rays $(\gtrsim 10 \mathrm{keV})$ are more likely to

\footnotetext{
2 a frequently updated list of X-ray SNe and references are available at:
} http://xray.astro.umass.edu/supernovae_list.htm] 
arise in the forward shock region. Because of the much higher density in the reverse shock region, the X-ray emission from this region will dominate that of the forward shock by the time that the expanding shell has become optically thin enough to allow the X-rays from the reverse shock region to reach the observer.

Both radio and X-ray emission are the result of the interaction of the ejecta with the CSM. The radio emission is believed to come from the region behind the circumstellar shock (see the Chap. by Chevalier \& Fransson and references therein), where the temperature is very high and the density low. In Fig. 1, we plot the $6 \mathrm{~cm}$ radio and soft X-ray band $(0.1-2.4 \mathrm{keV})$ light curves for several $\mathrm{SNe}$. Note that the radio emission is absorbed at early times (see below), but later on, the radio and X-ray emission are roughly proportional and show similar rates of decline.

The radio emission, in general, turns on in a late phase (months to years after the $\mathrm{SNe}$ ). Chevalier [10] proposed that the circumstellar shock generates the relativistic electrons and enhanced magnetic field necessary for synchrotron radio emission. The CSM, which is ionized by the UV produced during the outburst, absorbs this radio emission in the early phase. However, as the circumstellar shock moves outward, progressively less ionized material is left between the circumstellar shock and the observer, and absorption of the radio emission decreases. The observed radio flux density rises accordingly, first at high frequencies and subsequently (weeks to months later) at lower frequencies (cf, e.g. Fig. 2 in the Chap. by Sramek \& Weiler). At the same time, radio emission from the circumstellar shock region is decreasing slowly as the circumstellar shock expands. When radio absorption has become negligible, the radio light curve reflects this decline.

The signatures of circumstellar interaction in the radio, optical, and X-ray regimes have been found for a number of Type II SNe, such as the Type II-L SNe 1979C 77,79,19, 34 and 1980K [9,77,80,18,43. In the case of IIn $\mathrm{SNe}, \mathrm{H} \alpha$ typically exhibits a very narrow component (FWHM $\lesssim 200 \mathrm{~km} \mathrm{~s}^{-1}$ ) superimposed on a base of intermediate width (FWHM $\sim 1,000-2,000 \mathrm{~km} \mathrm{~s}^{-1}$ ). Sometimes a very broad component (FWHM $\sim 5,000-10,000 \mathrm{~km} \mathrm{~s}^{-1}$ ) is also present 21. The narrow optical lines are clear evidence for the presence of slowly moving dense circumstellar matter, probably photo-ionized by the intense flash of UV radiation. Examples of Type IIn SNe are SN 1986J [58,78,43,5, 33, SN 1995N 2, 45,74, and SN 1998S 22, 28, 46, 75, 54, Each of these SNe are discussed in more detail below.

Several nearby Type Ib/c SNe have been detected in the radio: SN 1990B [72], SN 1994I [73], SN 1997X 76], SN 1998bw [27]40] and SN 2002ap 3. SN 1994I [35]37, SN 1998bw 50,51] and SN 2002ap [57 have also been detected in Xrays. These SNe may be special cases - in particular, the strong evidence for association between SN 1998bw and GRB 980425 makes it unique (see the Chaps. by Galama and Frontera). 


\section{X-Ray Production Mechanism}

As discussed above, the most important process for the generation of a substantial amount of X-ray emission is the interaction of the ejecta with the CSM. However, depending on the age of the SNe, there can be other mechanism for the production of X-ray emission: (i) radio activity of the ejecta, (ii) an initial burst of X-rays during the break-out of the shock from the surface of the star, producing short time-scale $(\sim 1,000 \mathrm{sec})$ hard X-rays $(\sim 100 \mathrm{keV})$ and a very soft $(\sim 0.02 \mathrm{keV})$ black-body continuum, (iii) inverse Compton scattering of relativistic electrons with UV photons produced by the outburst, and (iv) pulsar-driven X-ray emission from the SN remnant in the case of a hot, fast spinning neutron star (if the latter is formed in the process of core collapse). This model is applicable to older SNRs, such as the Crab nebula.

Cases (ii)-(iv) will not be further discussed since they are not relevant to the $\mathrm{X}$-ray SNe reviewed in this chapter, and we will restrict ourselves to give a brief overview of the CSM interaction and radioactive decay.

\subsection{The Circumstellar Interaction}

The thermal X-ray luminosity, $L_{\mathrm{x}}$, produced by the shock heated CSM is the product of the emission measure, EM, and the cooling function, $\Lambda(T, Z, \Delta E)$, where $T$ is the CSM plasma temperature, $Z$ represents the elemental abundance distribution, and $\Delta E$ is the X-ray energy bandwidth. For spherically symmetric conditions $L_{\mathrm{x}}=\Lambda(T, Z, \Delta E) \mathrm{d} V n^{2}$, where $\mathrm{d} V$ is the volume, $n=\rho_{\mathrm{csm}} / \mathrm{m}$ is the number density of the shocked CSM and $m$ is the mean mass per particle $\left(2.1 \times 10^{-27} \mathrm{~kg}\right.$ for a $\mathrm{H}+\mathrm{He}$ plasma $)$. Assuming a constant supernova shock speed $v_{\mathrm{s}}, r=v_{\mathrm{s}} t$, where $t$ represents the time elapsed since the explosion. If the CSM density $\rho_{\mathrm{csm}}$ is dominated by a wind blown by the progenitor star of the supernova, the continuity equation requires $\dot{M}=4 \pi r^{2} \rho_{\mathrm{w}}(r) \times v_{\mathrm{w}}(r)$ through a sphere of radius $r$. After the SN shock plows through the CSM, its density is $\rho_{\mathrm{csm}}=4 \rho_{\mathrm{w}}$ [26]. We thus obtain $L_{\mathrm{x}}=4 /\left(\pi m^{2}\right) \Lambda(T) \times\left(\dot{M} / v_{\mathrm{w}}\right)^{2} \times\left(v_{\mathrm{s}} t\right)^{-1}$. We can hence use the observed X-ray luminosity at time $t$ after the outburst to measure the ratio $\dot{M} / v_{\mathrm{w}}$.

Each X-ray measurement at time $t$ is related to the corresponding distance $r$ from the site of the explosion. This site had been reached by the wind at a time depending on $v_{\mathrm{w}}$, or the age of the wind $t_{\mathrm{w}}=t v_{\mathrm{s}} / v_{\mathrm{w}}$. Usually, $v_{\mathrm{s}} \gg v_{\mathrm{w}}$ so that with $t$ only a few years we can look back quite a large time span in the evolution of the progenitor's wind and can use our measurements as a 'time machine' to probe the progenitor's history. Assuming that $v_{\mathrm{w}}$ did not change over $t_{\mathrm{w}}$, we can even directly measure the mass loss rate back in time. Integration of the massloss rate along the path of the expanding shell gives the mean density inside a sphere of radius $r$. For a constant wind velocity $v_{\mathrm{w}}$ and mass-loss rate $\dot{M}$, a $\rho_{\mathrm{csm}}=\rho_{0}\left(r / r_{0}\right)^{-s}$ profile with $s=2$ is expected.

After the expanding shell has become optically thin, it is expected that emission from the SN ejecta itself, heated by the reverse shock, dominates 
the X-ray output of the interaction regions due to its higher emission measure and higher density. For uniformly expanding ejecta the density structure is a function of its expansion velocity, $v$, and the time after the explosion, $t$ : $\rho_{\mathrm{sn}}=\rho_{0}\left(t / t_{0}\right)^{-3}\left(v / v_{0}\right)^{-n}$ with $\rho_{0}$ the ejecta density at time $t_{0}$ and velocity $v_{0}$ [26]. For a red supergiant progenitor, the power-law is rather steep with index $n \sim 20$ 26, 69. For constant $n$, the radius of the discontinuity surface between the forward and the reverse shock evolves in time $t$ with $r_{\mathrm{c}} \propto t^{m}$, where $m=(n-3) /(n-s)$ is the deceleration parameter.

\subsection{X-Ray Emission from Radioactive Decay}

The SN ejecta are radioactive. Gamma-rays due to the decay of $\mathrm{Ni}^{56}$ (half live 6.1 days) $\rightarrow \mathrm{Co}^{56}$ (half live 77 days) $\rightarrow \mathrm{Fe}^{56}$ and $\mathrm{Ni}^{57}$ (half live 36 hours) $\rightarrow$ $\mathrm{Co}^{57}$ (half live 270 days) $\rightarrow \mathrm{Fe}^{57}$ could, in principle, be detectable during the first few years after the SNe. The dominant lines produced in the decay of $\mathrm{Co}^{56}$ are $511 \mathrm{keV}$ (40\%), $847 \mathrm{keV}(100 \%), 1.04 \mathrm{MeV}(15 \%) 1.24 \mathrm{MeV}(66 \%), 1.76 \mathrm{MeV}$ (15\%), $2.02 \mathrm{MeV}$ (11\%), $2.60 \mathrm{MeV}(17 \%)$, and $3.25 \mathrm{MeV}(13 \%)$. The dominant lines produced in the decay of $\mathrm{Co}^{57}$ are $14 \mathrm{keV}(9 \%), 122 \mathrm{keV}(87 \%)$, and 136 $\mathrm{keV}(11 \%)$. The numbers in brackets represent the probablities that for each decaying nucleus the $\gamma$-ray in question is produced.

Several of these lines have been detected from SN 1987A (see the Chap. by McCray and references therein) but not from any other SN. This, of course, is not surprising given the very modest flux levels of the $\gamma$-rays and the large distances to all SNe. Hard ( $\gtrsim 10 \mathrm{keV})$ X-rays were observed from SN 1987A as early as 168-191 days after the outburst 68,16. At least part of these Xrays are believed to be the result of Compton scattering of $\gamma$-rays [41]. The light curves and spectral evolution of the X-rays and $\gamma$-rays are reasonably well understood (up to $\sim 300$ days after the outburst) if one assumes mixing of $\mathrm{Co}^{56}$ into the hydrogen rich envelope. The X-ray light curve at later times may be more problematic 42]. Whether a neutron star or a black hole was formed during the outburst of SN 1987A is still a matter of debate.

In case that a Type Ia SNe occurred within a distance of $\sim 50 \mathrm{kpc}$, one would also expect to see $\gamma$-rays from the radioactive by-products of the detonation (subsonical speed of the shock through the progenitor) or deflagration (supersonical speed) of the $\mathrm{C}+\mathrm{O}$ white dwarf. This, however, has not been observed as yet. 
Table 1. List of X-Ray Supernovae

\begin{tabular}{|c|c|c|c|c|c|c|c|c|}
\hline No. & $\begin{array}{l}\text { Supernova } \\
\text { Galaxy }\end{array}$ & Type & $\begin{array}{c}d \\
{[\mathrm{Mpc}]}\end{array}$ & $\begin{array}{l}t-t_{0}^{\star} \\
{[\text { days }]}\end{array}$ & $\begin{array}{c}f_{\mathrm{x}}^{\dagger} \\
{\left[10^{-14}\right]}\end{array}$ & $\begin{array}{c}L_{\mathrm{x}}^{\ddagger} \\
{\left[10^{38}\right]}\end{array}$ & Instrument & Ref. \\
\hline 1. & $\begin{array}{l}\text { SN 1978K } \\
\text { NGC } 1313\end{array}$ & II & 4.5 & 4,500 & $\begin{array}{l}204 \\
(0.1-2.4\end{array}$ & $\begin{array}{r}48 \\
\mathrm{keV})\end{array}$ & $\begin{array}{r}\text { ROSAT, ASCA } \\
\text { XMM-Newton }\end{array}$ & 49,61, \\
\hline 2. & $\begin{array}{l}\text { SN 1979C } \\
\text { NGC } 4321\end{array}$ & IIL & 17.1 & 5,900 & $\begin{array}{l}3.9 \\
(0.1-2.4\end{array}$ & $\begin{array}{r}14 \\
\mathrm{keV})\end{array}$ & $\begin{array}{r}\text { ROSAT, Chandra } \\
\text { XMM-Newton }\end{array}$ & 35 \\
\hline 3. & $\begin{array}{l}\text { SN 1980K } \\
\text { NGC } 6946\end{array}$ & IIL & 5.1 & 35 & $\begin{array}{l}1.5 \\
(0.1-2.4\end{array}$ & $\begin{array}{r}0.5 \\
\mathrm{keV})\end{array}$ & Einstein, ROSAT & \\
\hline 4. & $\begin{array}{l}\text { SN 1986J } \\
\text { NGC } 891\end{array}$ & IIn & 9.6 & 3,300 & $\begin{array}{l}119 \\
(0.1-2.4\end{array}$ & $\begin{array}{r}140 \\
\mathrm{keV})\end{array}$ & ROSAT, ASCA & 目, 12 \\
\hline 5. & $\begin{array}{l}\text { SN 1987A } \\
\text { LMC }\end{array}$ & IIP & 0.05 & - & - & - & $\begin{array}{r}\text { ROSAT, Chandra } \\
\text { XMM-Newton }\end{array}$ & 目, 15 \\
\hline 6. & $\begin{array}{l}\text { SN 1988Z } \\
\text { MCG+03-28-02 }\end{array}$ & $22^{\text {IIn }}$ & 89 & 2,370 & $\begin{array}{l}0.9 \\
(0.1-2.4\end{array}$ & $\begin{array}{r}110 \\
\mathrm{keV})\end{array}$ & ROSAT & \\
\hline 7. & $\begin{array}{l}\text { SN 1993J } \\
\text { NGC } 3031\end{array}$ & IIb & 3.6 & 6 & $\begin{array}{l}130 \\
(0.5-2\end{array}$ & $\begin{array}{r}20 \\
\mathrm{keV})\end{array}$ & ROSAT, ASCA & 36 . \\
\hline 8. & $\begin{array}{l}\text { SN 1994I } \\
\text { NGC } 5194\end{array}$ & Ic & 7.7 & 79 & $\begin{array}{l}2.3 \\
(0.3-2\end{array}$ & $\begin{array}{c}1.6 \\
\mathrm{keV})\end{array}$ & ROSAT, Chandra & 3537 \\
\hline 9. & $\begin{array}{l}\text { SN 1994W } \\
\text { NGC } 4041\end{array}$ & IIP & 25 & 1,180 & $\begin{array}{c}11 \\
(0.1-2.4\end{array}$ & $\begin{array}{r}85 \\
\mathrm{keV})\end{array}$ & ROSAT & \\
\hline 10. & $\begin{array}{l}\text { SN 1995N } \\
\text { MCG-2-38-017 }\end{array}$ & IIn & 24 & 440 & $\begin{array}{c}40 \\
(0.1-2.4\end{array}$ & $\begin{array}{r}175 \\
\mathrm{keV})\end{array}$ & ROSAT, ASCA & \\
\hline 11. & $\begin{array}{l}\text { SN 1998bw } \\
\text { ESO 184-G82 }\end{array}$ & Ic & 38 & 0.4 & $\begin{array}{l}23-40 \\
\quad(2-10\end{array}$ & $\begin{array}{l}40-70 \\
\mathrm{keV})\end{array}$ & Beppo-Sax & 5051 \\
\hline 12. & $\begin{array}{l}\text { SN 1998S } \\
\text { NGC } 3877\end{array}$ & IIn & 17 & 64 & $\begin{array}{l}27 \\
(2-10\end{array}$ & $\begin{array}{r}92 \\
\mathrm{keV})\end{array}$ & $\begin{array}{r}\text { Chandra } \\
X M M-N e w t o n\end{array}$ & 54 \\
\hline 13. & $\begin{array}{l}\text { SN 1999em } \\
\text { NGC } 1637\end{array}$ & IIP & 7.8 & 4 & $\begin{array}{l}1.0 \\
(2-10\end{array}$ & $\begin{array}{l}0.7 \\
\mathrm{keV})\end{array}$ & Chandra & 54 \\
\hline 14. & $\begin{array}{l}\text { SN 1999gi } \\
\text { NGC } 3184\end{array}$ & IIP & 8.7 & 29 & $\begin{array}{l}0.1 \\
(0.5-10\end{array}$ & $\begin{array}{r}0.1 \\
\mathrm{keV})\end{array}$ & Chandra & 65 \\
\hline 15. & $\begin{array}{l}\text { SN 2002ap } \\
\text { NGC } 628\end{array}$ & Ic & 10 & 4 & $\begin{array}{l}0.2 \\
(0.1-10\end{array}$ & $\begin{array}{r}0.2 \\
\mathrm{keV})\end{array}$ & XMM-Newton & 57 \\
\hline$\S$ & $\begin{array}{l}\text { maximum obser } \\
\text { maximum obser } \\
\text { X-ray flux still }\end{array}$ & $\begin{array}{l}\text { rved X } \\
\text { rved X } \\
\text { rising }\end{array}$ & lu & uni & 10 & $\mathrm{Cl}$ & $\begin{array}{l}\text { X-ray flux } \\
\mathrm{s}_{-1}^{-1}\end{array}$ & \\
\hline
\end{tabular}



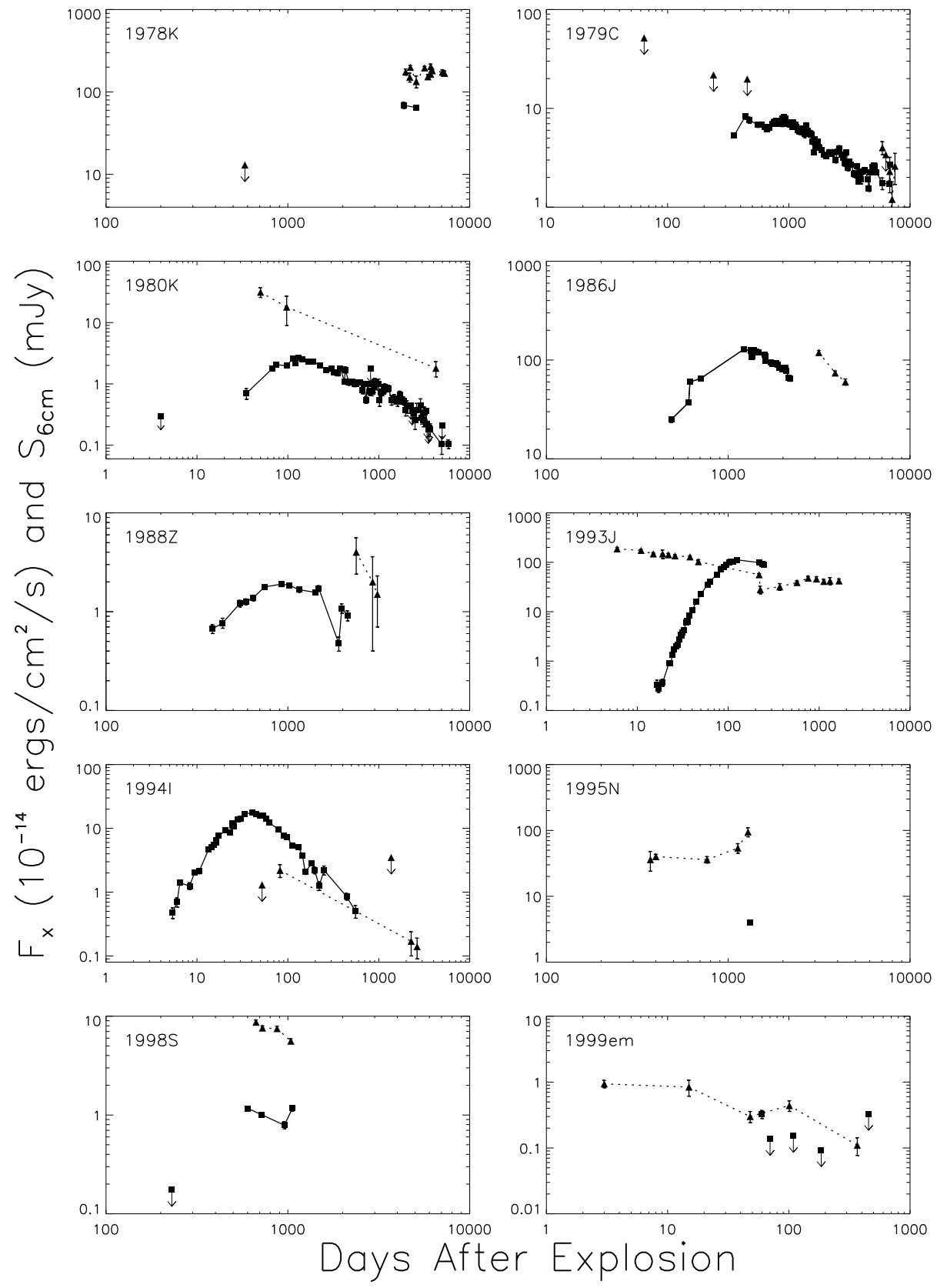

Fig. 1. $6 \mathrm{~cm}$ radio (VLA and ATCA; filled boxes, solid lines) and soft X-ray band lightcurves (0.2-2.4 keV; triangles and dashed lines) of nine Type II and one Ic SNe. 


\section{Overview of X-ray Supernovae}

In the following section we will give a brief overview of X-ray observations of all X-ray SNe except SN 1987A, which is discussed in detail in the Chap. by McCray.

\subsection{SN 1978K in NGC 1313}

Due to its discovery in the early phase of the ROSAT mission and the relatively high flux level, SN 1978K is one of the best studied SN in the X-ray regime. While an Einstein observation $\sim 1.5$ years after the outburst only provided an upper limit to the soft $(0.5-2 \mathrm{keV})$ band X-ray luminosity $\left(L_{\mathrm{x}} \lesssim 2 \times 10^{38} \mathrm{ergs} \mathrm{s}^{-1}[62]\right)$, SN $1978 \mathrm{~K}$ has been successfully monitored with ROSAT on 13 different dates ranging from $\sim 12$ to 20 years after the outburst 6263. During this period, the SN showed no apparent evolution in X-ray flux $\left(L_{\mathrm{x}} \sim 4 \times 10^{39} \mathrm{ergs} \mathrm{s}^{-1}\right)$. $A S C A$ GIS/SIS and ROSAT PSPC observations provided the first broad-band (0.1-10 keV), medium resolution X-ray spectra of a young SN [49]. The spectra showed no emission lines and could be equally well described by either a thermal bremsstrahlung spectrum or thermal plasma emission with a temperature of $\sim 3 \mathrm{keV}$ and sub-solar abundances $\left(Z \sim 0.2 Z_{\odot}\right)$. Alternatively, the spectrum could be described by a power with photon index $\Gamma \sim 2.2$ and an absorbing column of $N_{\mathrm{H}} \sim 1 \times 10^{21} \mathrm{~cm}^{-2}$. From the X-ray lightcurve the index $n$ for the ejecta power-law distribution $\rho_{\text {ejecta }} \propto r^{-n}$ could be constrained to be in the range 4-12 for an assumed CSM density gradient $\rho_{\mathrm{csm}} \propto r^{-s}$ with index $s=1.5-2$ [63]. Estimates for the mass-loss rate are $\dot{M} \sim 1 \times 10^{-4} M_{\odot} \mathrm{yr}^{-1}$, as is expected for a massive progenitor. A recent XMM-Newton observation showed that SN $1978 \mathrm{~K}$ is still at the same flux level as observed during the last $\sim 22$ years and shows no apparent evolution $\left(L_{\mathrm{x}}=4 \times 10^{39} \mathrm{ergs} \mathrm{s}^{-1}\right.$ [38]). The superior photon collecting area and spectral resolution of the XMM-Newton instruments with a total of $\sim 20,000$ net counts from SN 1978K in the combined EPIC-pn and MOS spectra demonstrates the dramatic technological progress that has been made in the development of X-ray instruments over the last two decades. The best-fit model to the spectrum gave a two-temperature thermal emission component with $k T_{\text {low }} \sim 0.8 \mathrm{keV}$ and $k T_{\text {high }} \sim 3 \mathrm{keV}$. The XMM-Newton data clearly show, for the first time, emission from the forward (high temperature component) and reverse shock region (low temperature component).

\section{$3.2 \quad \mathrm{SN}$ 1979C in NGC 4321 (M100)}

An X-ray source was discovered in a ROSAT HRI observation of M100 at the position of SN 1979C $\sim 16$ years after the outburst (see Fig. 2), with a (0.1$2.4 \mathrm{keV}$ ) luminosity of $L_{\mathrm{x}}=1.3 \times 10^{39} \mathrm{ergs} \mathrm{s}^{-1}$ 34, and in a ROSAT HRI follow-up observation $\sim 19$ years after the outburst. For three earlier Einstein observations, taken on days 64,239 and 454 after the outburst, only $3 \sigma$ upper limits could be established $\left(1.8 \times 10^{40} \mathrm{ergs} \mathrm{s}^{-1}, 7.6 \times 10^{39} \mathrm{ergs} \mathrm{s}^{-1}\right.$ and $6.9 \times$ $10^{39} \mathrm{ergs} \mathrm{s}^{-1}$, respectively). The ROSAT data imply a mass-loss rate of $\dot{M} \sim$ 


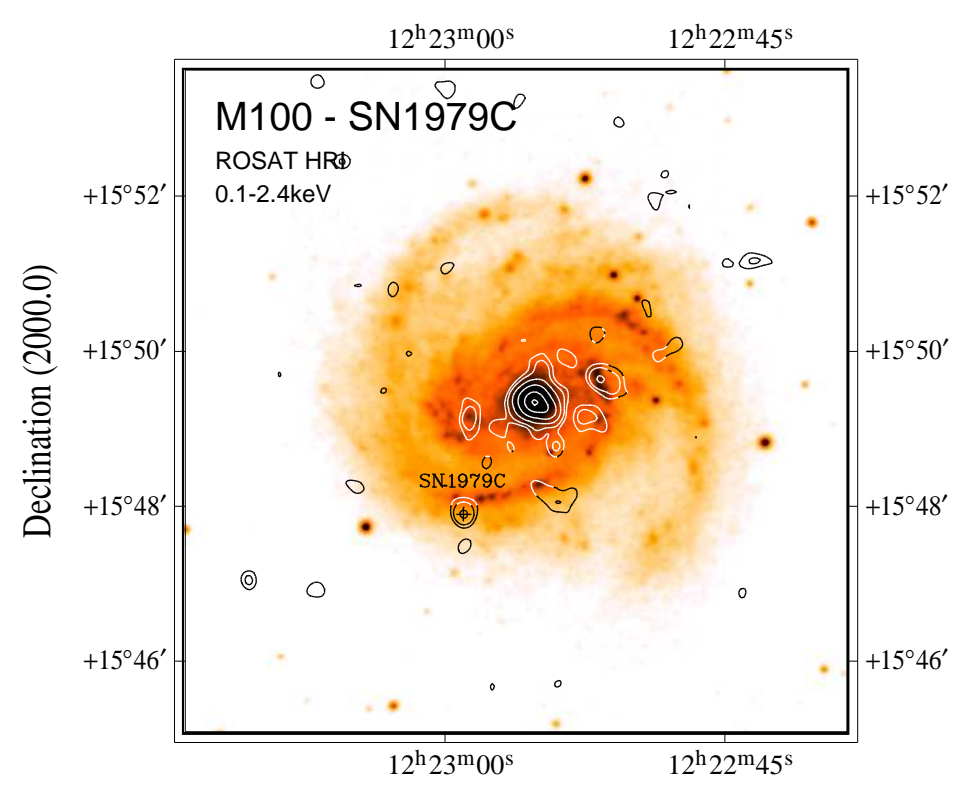

Right Ascension (2000.0)

Fig. 2. ROSAT HRI soft (0.1-2.4 keV) band X-ray contours of SN 1979C in M100, overlayed onto an optical image.

$1 \times 10^{-4} M_{\odot} \mathrm{yr}^{-1}$, similar to mass-loss rates of other massive SN progenitors (e.g. SNe 1978K, 1986J, 1988Z and 1998S) and in agreement with the mass-loss rate inferred from VLA radio observations [79]. Since neither a ROSAT PSPC 'last light' observation (requested by the author), nor ASCA and Chandra pointings [39.55] gave enough photon statistics to constrain the spectral properties, no information is available about the spectral characteristics of the X-ray emission. Both the ROSAT PSPC and Chandra observations, however, showed that the emission is rather soft $(<2 \mathrm{keV})$ and likely originates from shock-heated material in the reverse shock. The combined ROSAT, ASCA and Chandra data indicate a rather slow X-ray rate of decline (decrease from $L_{\mathrm{x}}=1.3 \times 10^{39} \mathrm{ergs} \mathrm{s}^{-1}$ to $0.9 \times 10^{39} \mathrm{ergs} \mathrm{s}^{-1}$ over 4.3 years in the $0.1-2.4 \mathrm{keV}$ band [39]) consistent with the rate of decline $\left(t^{-0.7}\right)$ observed in the radio regime over the first $\sim 10$ years after the outburst [79. Since both the X-ray and radio emission processes are considered to be linked to the interaction of the SN shock with the high-density envelope of matter (see Sec. 1), one would expected the X-ray evolution to follow the observed radio evolution. 


\section{3 $\quad$ SN 1980K in NGC 6946}

Historically, the IIL SN 1980K in NGC 6946 was the first SN discovered in X-rays [9]. SN 1980K was detected in an Einstein IPC observation with a $(0.2-4 \mathrm{keV})$ luminosity of $L_{\mathrm{x}}=4.8 \times 10^{38} \mathrm{ergs} \mathrm{s}^{-1} 35$ days after the outburst [9, 61. A followup observation on day 82 showed that the X-ray source has faded by a factor of $\sim 5$. Despite the rather large IPC error box of $\sim 1^{\prime}$, the positional coincidence of the X-ray source with the optical position of the SN was later confirmed with the ROSAT PSPC 60. Spectral analysis of the Einstein and ROSAT data showed that the emission was rather soft (Einstein: $k T \sim 0.5 \mathrm{keV}$ [9]; ROSAT: $k T \sim 0.4 \mathrm{keV}$ [61]) and in relatively good agreement with theoretical predictions $(k T \sim 0.7 \mathrm{keV}$ [11). Given the low photon statistics, physical processes as to the production of the X-rays could not be constrained. The data, however, could be successfully used to give estimates as to the mass loss rate of the SN progenitor $\left(\dot{M} \sim 0.5-5 \times 10^{-6} M_{\odot} \mathrm{yr}^{-1}\right)$ and to demonstrate that SNe do not significantly contribute to the overall diffuse X-ray background.

\subsection{SN 1986J in NGC 891}

SN 1986J was observed with the ROSAT HRI and with ASCA over a period covering $\sim 9-13$ years after the outburst [5, 12, 33]. Contrary to many other Xray SNe (e.g. SNe 1978K, 1993J, 1994I and 1998S), SN 1986J showed a rather fast X-ray rate of decline $\left(L_{\mathrm{x}} \propto t^{-2}[33]\right)$. Two ASCA spectra indicated that the emission was rather hard $(k T=5-7 \mathrm{keV})$ compared to other X-ray SNe (cf. Sec. 3.3 and 3.2). The ASCA spectra also clearly showed a Fe-K emission line at $6.7 \mathrm{keV}$ with a width of $<20,000 \mathrm{~km} \mathrm{~s}^{-1}$ (FWHM). The spectral properties and rate of decline were used to test two different models that could account for the observed X-ray emission: in the CSM interaction model the X-ray emission is thought to originate in the reverse shock running through the outer layers of the SN ejecta [11]. While both the predicted X-ray luminosity $\left(\sim 10^{40} \operatorname{ergs~s}^{-1}\right)$, mass-loss rate $\left(\sim 10^{-4} M_{\odot} \mathrm{yr}^{-1}\right.$ for an assumed wind velocity of $\left.10 \mathrm{~km} \mathrm{~s}^{-1}\right)$ and line width for the shocked plasma $\left(\sim 10,000 \mathrm{~km} \mathrm{~s}^{-1} \mathrm{FWHM}\right)$ are in agreement with the X-ray data, the CSM model predicts a temperature significantly lower $(\sim 1 \mathrm{keV})$ than observed. An alternative model proposed by Chugai [12] assumes that the emission arises from shocked interstellar clumps after the forward shock plows through the CSM. This model predicts a higher temperature of the shocked regions which are reconciled by the data, and a similar mass-loss rate for the progenitor. Potential problems, however, arise in the fact that the shocked clouds should have narrow emission lines (some $\times 100 \mathrm{~km} \mathrm{~s}^{-1}$ ). While narrow emission lines have in fact been observed in optical spectra of SN 1986J (e.g. H $\alpha$ emission

lines with a FWHM of $530 \mathrm{~km} \mathrm{~s}^{-1}[58[3]$ ), questions arise as to how the clouds can account for both the optical and X-ray emission, which require a shock velocity of approx. $2,000 \mathrm{~km} \mathrm{~s}^{-1}$ for the observed temperature [33]. 


\subsection{SN 1988Z in MCG+03-28-011}

At a distance of $\sim 89 \mathrm{Mpc}$ (assuming $H_{0}=75 \mathrm{~km} \mathrm{~s}^{-1} \mathrm{Mpc}^{-1}$ ), SN $1998 \mathrm{Z}$ is the most distant and most luminous X-ray $\left(L_{\mathrm{x}} \sim 10^{41}\right.$ ergs s$^{-1} 6.5$ years after the outburst) and radio $\mathrm{SN}\left(L_{6 \mathrm{~cm}} \sim 9 \times 10^{32} \mathrm{ergs} \mathrm{s}^{-1} \mathrm{~Hz}^{-1}\right.$ on day 1,253) discovered to date 17,72. Given the observed X-ray luminosity, a CSM density of $\sim 10^{6} \mathrm{~cm}^{-3}$ was inferred at a radius of $r=5 \times 10^{16} \mathrm{~cm}$ from the site of the explosion (corresponding to the date of the X-ray observation) [17]. The radio data further implied that the dense cocoon resulted from a high mass-loss rate of the progenitor of $\dot{M} \sim 10^{-4} M_{\odot} \mathrm{yr}^{-1}$ in the late stage of the massive (20-30 $\left.M_{\odot}\right)$ progenitor [72. Two ROSAT HRI follow-up observations [1] showed that the SN rapidly faded by a factor of $\sim 5$ within 2 years (see Fig. 11).

\subsection{SN 1993J in NGC 3031 (M81)}

ROSAT observations of SN 1993J gave strong observational support for models that attribute the X-ray emission to the interaction of an outgoing and reverse shock with the CSM. Whereas the early X-ray spectrum on day 6 was hard $\left(T \sim 10^{8.5} \mathrm{~K}\right)$ and absorbed by the Galactic foreground column only $\left(N_{\mathrm{H}} \sim 4 \times 10^{20} \mathrm{~cm}^{-2}\right)$, a softer component $\left(T \sim 10^{7} \mathrm{~K}\right)$ dominated at day $\sim 200$ [26]. ASCA data from day 8 in the broader 1-10 keV X-ray band were also best characterized by hard $\left(\sim 10^{8} \mathrm{~K}\right)$ thermal emission $[70$. Very hard Xray photons were recorded during days 9-15 and 23-36 using OSSE onboard the Compton Gamma-Ray Observatory. During these dates, SN 1993J reached (50$150 \mathrm{keV}$ band) luminosities of $L_{\mathrm{x}}=5.5 \times 10^{40} \mathrm{ergs} \mathrm{s}^{-1}$ and $3.0 \times 10^{40} \mathrm{ergs} \mathrm{s}^{-1}$, respectively, before falling below the OSSE detection threshold during a longer observation on days 93-121 [44]. The harder component is due to the circumstellar shock region, whereas the softer component must have come from the region behind the reverse shock. The emergence of this radiation could be attributed to the decreased absorption by a cool shell 26 . The early $(<200$ days) ROSAT and ASCA data were successfully used to estimate the mass-loss rate of the SN 1993J progenitor $\left(\dot{M} \sim 4 \times 10^{-5} \mathrm{M}_{\odot} \mathrm{yr}^{-1}\right)$ and indicate that the CSM density profile might be flatter $\left(\rho_{\mathrm{csm}} \propto r^{-s}\right.$ with $\left.1.5 \lesssim s \lesssim 1.7\right)$ than expected for spherically symmetric conditions $(s=2)$ [26].

Intriguing new results came form the analysis of the entire ROSAT data set, covering a period from 6 days to 7 years after the outburst of SN 1993J. The combined ROSAT PSPC and HRI lightcurve is best fitted by a $L_{\mathrm{x}} \propto t^{-0.27}$ $\mathrm{X}$-ray rate of decline (cf. Fig. 1). Since each X-ray observation at time $t$ is related to the corresponding distance $r$ from the site of the explosion, $r=v_{\mathrm{s}} \times t$ (with shock front velocity $v_{\mathrm{s}}$ ), and to the age of the stellar wind, $t_{\mathrm{w}}=t v_{\mathrm{s}} / v_{\mathrm{w}}$, the ROSAT measurements could be used as a 'time machine' to look back into the history of the stellar wind. During the observed period, the SN shell has reached a radius of $3 \times 10^{17} \mathrm{~cm}$ from the site of the explosion, corresponding to $\sim 10^{4}$ years in the progenitors stellar wind history. Contrary to an expected CSM density profile of $\rho_{\mathrm{csm}}=\rho_{0}\left(r / r_{0}\right)^{-s}$ profile with index $s=2$ for a constant wind velocity $v_{\mathrm{w}}$ and mass-loss rate $\dot{M}$, the data revealed a significantly flatter 


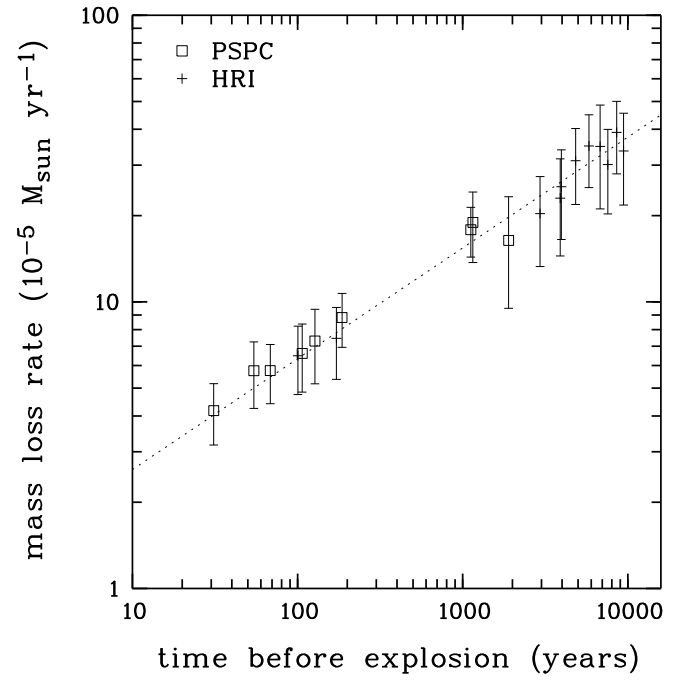

Fig. 3. Mass-loss rate history of the SN 1993J progenitor. ROSAT PSPC data are marked by boxes, HRI data are indicated by crosses 36 .

profile of $\rho_{\mathrm{csm}} \propto r^{-1.63}$. After ruling out alternative scenario that might explain the data (e.g. variations in velocity and/or temperature of the shocked CSM and a non-spherical geometry caused by a binary evolution of the progenitor) it was concluded that the mass-loss rate of the progenitor has decreased constantly from $\dot{M}=4 \times 10^{-4}$ to $4 \times 10^{-5} \mathrm{M}_{\odot} \mathrm{yr}^{-1}\left(v_{\mathrm{w}} / 10 \mathrm{~km} \mathrm{~s}^{-1}\right)$. The observed evolution clearly reflects either a decrease in the mass-loss rate, an increase in the wind speed or a combination of both, indicating that the progenitor likely was making a transition from the red to the blue supergiant phase during the late stage of its evolution. This scenario for the evolution of the SN 1993J progenitor has interesting similarities with that of SN 1987A, whose progenitor (aka SK-69 202) completely entered the blue supergiant phase after significant mass-transfer to a companion some $\times 10^{4}$ years prior to the explosion (see 25,53] and the Chap. by McCray).

\subsection{SN 1994I in NGC 5194 (M51)}

SN 1994I represents a unique case since it is the first Ic SN which has been detected in soft X-rays. Based on early ROSAT HRI observations, evidence for soft (0.1-2.4 keV) X-ray emission was found on day 83 after the outburst 35. However, due to the spatial resolution of the HRI ( $5^{\prime \prime}$ on-axis) and the close location to the X-ray bright nucleus of M51 (18" offset), as well as the high level of diffuse X-ray emission in the bulge of M51, the results were not entirely conclusive. Two follow-up observations 6-7 years after the outburst, using the superior capabilities of Chandra in terms of spatial resolution ( $0 . .5$ on-axis) and sensitivity revealed an X-ray source consistent with the radio position of the SN 


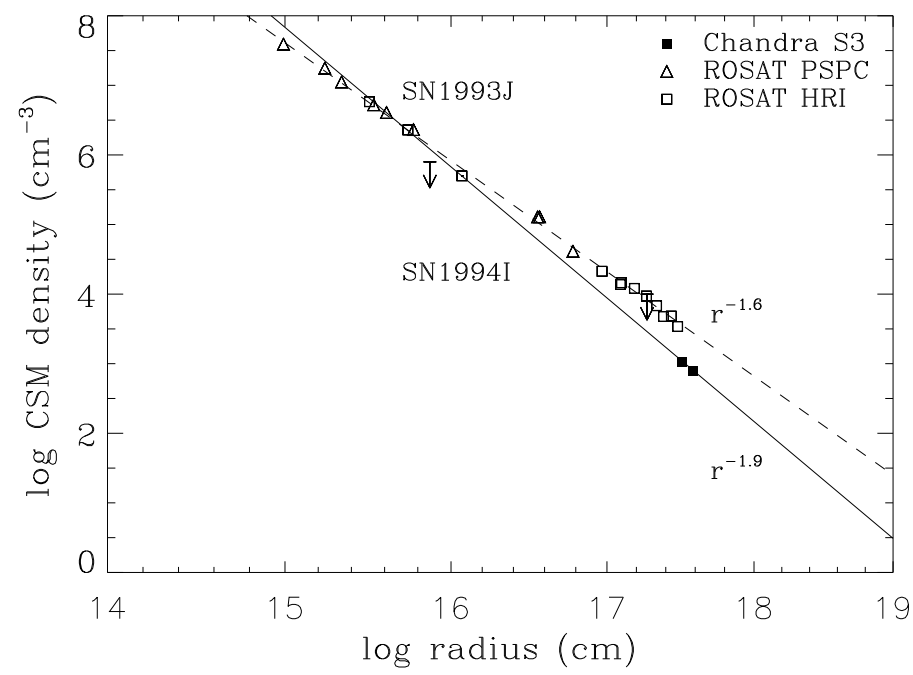

Fig. 4. CSM density profiles of SNe 1994I and 1993J as a function of shell expansion radius 37 .

(0.5 offset 37]). SN 1994I was detected with Chandra at a $\sim 6 \sigma$ level with a luminosity of $L_{\mathrm{x}} \sim 1 \times 10^{37} \mathrm{ergs} \mathrm{s}^{-1}$ in the $0.3-2 \mathrm{keV}$ band. Combined with the earlier ROSAT data, the X-ray lightcurve was parameterized as $L_{\mathrm{x}} \propto(t-$ $\left.t_{0}\right)^{-s} \times e^{-\tau}$ with $\tau \propto\left(t-t_{0}\right)^{-\beta}$, where the external absorption of the emission at early times is represented by the $e^{-\tau}$ term ('optical depth') with a subsequent exponential rate of decline with index $s$. A best-fit X-ray rate of decline of $L_{\mathrm{x}} \propto t^{-1.4}$ was found with index $\beta=1$ for the optical depth. The index $s$ is in good agreement with the radio rate of decline of SN 1994I $(s=1.6)$. Interestingly, similarly fast rates of decline have been inferred for other Ib/c SNe as well (e.g. $s=1.4$ for SN 1998bw inferred from the tentative hard X-ray band Beppo-Sax detection; $s=1.3,1.6$ and 1.5, respectively, for the radio SNe 1990B $1983 \mathrm{~N}$ and 1984L). Assuming that the X-ray emission is due to the shocked CSM, a massloss rate of $\dot{M} \sim 1 \times 10^{-5} M_{\odot} \mathrm{yr}^{-1}\left(v_{\mathrm{w}} / 10 \mathrm{~km}^{-1}\right)$ consistent with all ROSAT and Chandra detections on days 82, 2,271 and 2,639 and a ROSAT upper limit on day 1,368 after the outburst was derived. Furthermore, the CSM density profile was constructed for the different radii corresponding to the dates of the observations. The combined ROSAT and Chandra data gave a best-fit CSM profile of $\rho_{\mathrm{csm}} \propto r^{-(1.9 \pm 0.1)}$ for $r=0.1-3.8 \times 10^{17} \mathrm{~cm}$, consistent with what is expected for a constant stellar wind velocity and constant mass-loss rate of the progenitor $\left(r^{-2}\right)$. A comparison of the CSM profiles of SNe 1994I and 1993J, the only SNe for which the CSM profile was ever constructed [36], is illustrated in Fig. 4. 


\section{8 $\mathrm{SN}$ 1994W in NGC 4041}

A ROSAT HRI observation of SN 1994W was performed 1,180 days after the outburst 64]. An X-ray source, with a luminosity of $L_{\mathrm{x}} \sim 8 \times 10^{39} \mathrm{ergs} \mathrm{s}^{-1}$, was found to coincide with the position of SN 1994W (offset 1".4). Given the positional coincidence, the low probability of $\sim 3 \times 10^{-3}$ of chance coincidence with a background or foreground object, and the indications of CSM interaction based on the narrow absorption lines in the optical spectra made a detection of SN 1994W in X-rays likely. However, since there are no other X-ray observations of the host galaxy NGC 4041, confirmation of the detection is still pending. Also, since SN 1994W is the only X-ray SN which has not been detected in the radio, no additional information about the CSM interaction is available. Interestingly, a CSM number density of $\rho_{\mathrm{csm}} \gtrsim 10^{8} \mathrm{~cm}^{-3}$ was inferred from the optically thick FeII lines $\left[67\right.$ at radii of $8.5 \times 10^{14} \mathrm{~cm}$ and $1.1 \times 10^{15} \mathrm{~cm}$ (corresponding to days 31 and 57 after the outburst). Based on the PCygni $\mathrm{H} \alpha$ and $\mathrm{H} \beta$ line profiles of SN 1997ab (Type IIn), a similar CSM density of $\sim 10^{8} \mathrm{~cm}^{-3}$ at $\sim 10^{15} \mathrm{~cm}$ was estimated [59]. The results are in remarkable agreement with the CSM number density of $\sim 10^{7.5}-10^{8} \mathrm{~cm}^{-3}$ for SNe 1994I and 1993J at $r \sim 10^{15} \mathrm{~cm}$ (cf. Fig. (1) and appears to be a common CSM density for massive progenitors ( $\gtrsim 10 M_{\odot}$ ZAMS) at this distance from the site of the explosion.

\subsection{SN 1995N in MCG-2-38-017}

SN $1995 \mathrm{~N}$ was observed with the ROSAT HRI $\sim 1$ and 2 years after the outburst, followed by an ASCA observation some months later [24]. The ASCA spectrum could be well described by a $k T \sim 10 \mathrm{keV}$ thermal bremsstrahlung spectrum or a power-law with photon index $\sim 1.7$, both absorbed by the Galactic foreground column only $\left(N_{\mathrm{H}} \sim 10^{21} \mathrm{~cm}^{-2}\right)$. Assuming that the spectrum has not changed during the observed period, SN $1995 \mathrm{~N}$ has faded by approx. $30 \%$ during the two ROSAT intervals and subsequently brightened by a factor of two (ASCA). Together with the high observed luminosity $\left(\sim 10^{41} \mathrm{ergs} \mathrm{s}^{-1}\right)$, this might be indicative that the SN shock has plowed through a dense and inhomogeneous CSM.

\subsection{SN 1998bw in ESO 184-G82}

Hard $(2-10 \mathrm{keV}) \mathrm{X}$-ray emission was found with Beppo-Sax from the position of the unusual SN 1998bw which might be associated with a $\gamma$-ray burst event (aka GRB 980425 [50,51]). The detection, however, is still tentative due to the large error box of the Beppo-Sax Wide Field Camera (between $3^{\prime}$ and $8^{\prime}, 90 \%$ confidence limit) and the large probability of $\sim 60 \%$ for a random chance coincidence. Due to the likely association of SN 1998bw with GRB 980425, we refer to the Chaps. by Galama and Frontera for a more detailed review of the observations. 


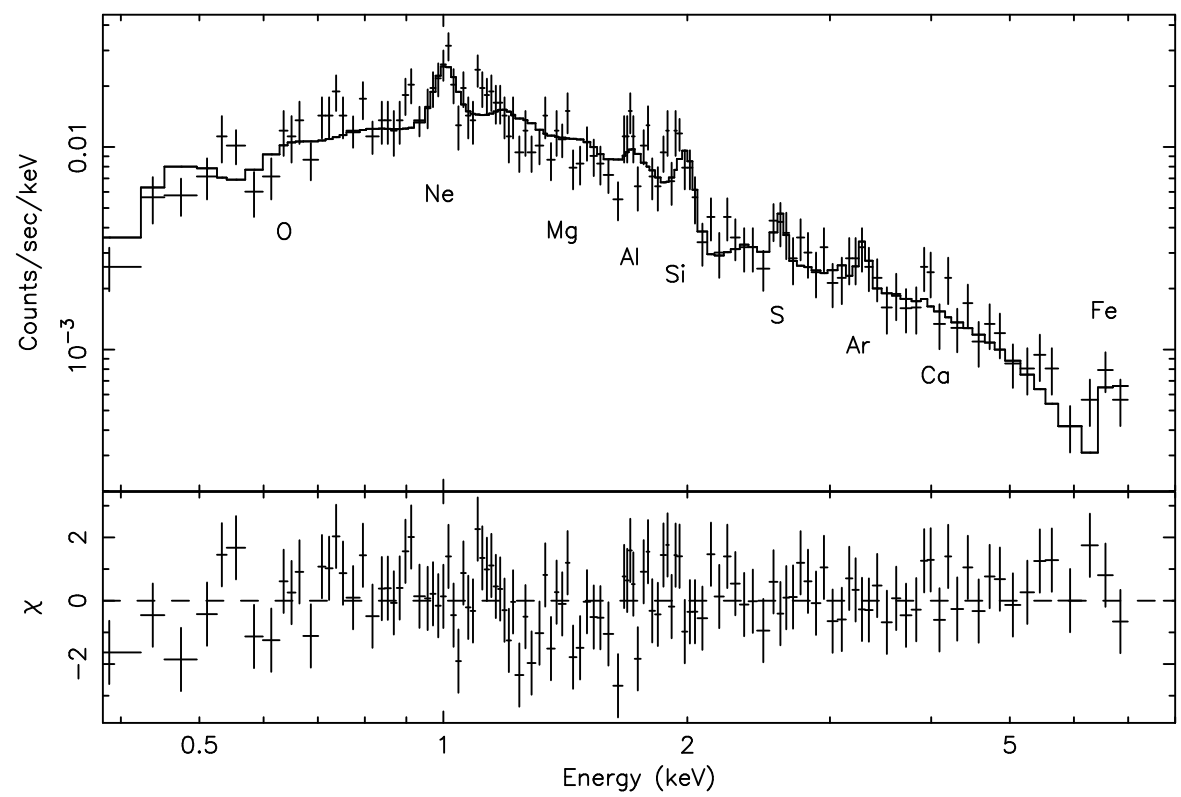

Fig. 5. Chandra ACIS-S3 spectrum of SN 1998S [54]. The solid line gives the best fit model to the data. Labels indicate the location of emission lines (see Sec. 3.11).

\subsection{SN 1998S in NGC 3877}

A wealth of X-ray data has been collected for the Type IIn SN 1998S with Chandra and XMM-Newton [54,38. At an age of $\sim 2-3$ years after the outburst, SN 1998S is still bright in X-rays (Chandra data on day 668: $L_{\mathrm{x}} \sim$ $9 \times 10^{39} \mathrm{ergs} \mathrm{s}^{-1}$ [54]; XMM-Newton data on day 1,202: $L_{\mathrm{x}} \sim 3 \times 10^{39} \mathrm{ergs} \mathrm{s}^{-1} ; 2$ $10 \mathrm{keV}$; [38]) and increasing in cm radio flux density. The X-ray lightcurve based on five Chandra and one XMM-Newton observation is best described by an Xray rate of decline of $L_{\mathrm{x}} \propto t^{-1.3}$. The inferred mass-loss rate is $\dot{M} \sim 2 \times 10^{-4} M_{\odot}$ $\mathrm{yr}^{-1}$. Spectral analysis of the Chandra data show a best-fit temperature of $k T \sim 10 \mathrm{keV}$ and high over-abundance of heavy elements, such as $\mathrm{Ne}, \mathrm{Al}, \mathrm{Si}$, $\mathrm{S}, \mathrm{Ar}$ and $\mathrm{Fe}$ (3-30 over solar). The Chandra spectrum and best-fit model are shown in Fig. 5 . The high-quality spectra, in combination with theoretical modeling, allowed, for the first time, to use emission line ratios of elements produced before the core collapse and during the explosion to constrain the progenitor's mass. The observed $\mathrm{O} / \mathrm{Si}$ ratio of $1-12, \mathrm{Mg} / \mathrm{Si}$ of $0-0.7$ and $\mathrm{Ne} / \mathrm{Si}$ of $0.6-14$ led to an estimate of the progenitor's mass of $\sim 18 M_{\odot}$ [54]. A more recent XMMNewton observation revealed the emergence of a soft $(\sim 0.8 \mathrm{keV})$ component from the reverse shock in addition to the harder component $(\sim 9 \mathrm{keV})$ already observed with Chandra, and a prominent and broad Si emission line at $1.89 \mathrm{keV}$ with an equivalent width of $\sim 50 \mathrm{eV}$ (FWHM) 38]. 


\subsection{SN 1999em in NGC 1637}

SN 1999em is the only IIP SN detected both in the radio and X-rays, although at a very low level $\left(L_{6 \mathrm{~cm}}=2.2 \times 10^{25} \mathrm{ergs} \mathrm{s}^{-1} \mathrm{~Hz}^{-1}\right.$ on day $34 ; L_{\mathrm{x}}=7 \times$ $10^{37} \mathrm{ergs} \mathrm{s}^{-1}$ on day 4 [54]). The Chandra X-ray data indicated a non-radiative interaction of the the SN ejecta with the CSM, and a low mass-loss rate of $\dot{M} \sim$ $2 \times 10^{-6} M_{\odot} \mathrm{yr}^{-1}$. Five Chandra observations, performed on days 4-368 after the outburst, showed a temperature evolution during this period, with a softening of the emission from originally $\sim 5 \mathrm{keV}$ to $\sim 1 \mathrm{keV}$ for an assumed thermal bremsstrahlung spectrum. This evolution confirmed theoretical predictions of a change in temperature and indicate a rather flat density profile of the ejecta. Given an observed evolution of $L_{\mathrm{x}} \propto t^{-1}$, the SN was below the detection limit during observations performed on days 495 and 633 after the outburst 54].

\subsection{SN 1999gi in NGC 3184}

SN 1999gi was detected in two Chandra observations on days 29 and 54 after the outburst 65. Similarly to SNe 1987A and 1999em [54, SN 1999gi only reached a relatively low luminosity $\left(\sim 10^{37} \mathrm{ergs} \mathrm{s}^{-1} ; 0.5-10 \mathrm{keV}\right.$ band), implying a low mass-loss rate of $\dot{M} \sim 10^{-6} M_{\odot} \mathrm{yr}^{-1}$. Since these SNe are of Type IIP, it has been suggested that IIP SNe explode in a low-density CSM environment 65]. By contrast, progenitors of IIL (e.g. SN 1979C, 1980K), IIb (SN 1996J) and IIn (SN 1986J, 1988Z, 1995N, 1998S) SNe can deposit a substantial amount of CSM prior to the explosion. An interesting argument was put forward by Chugai 13], who described the mass-loss rate as a function of the progenitor's zero-age main sequence star mass (ZAMS). In this scenario the mass-loss rate rises to a peak near $\sim 10 M_{\odot}$ and falls with increasing mass, leading to a IInIIL-IIP sequence. Although the X-ray and radio data collected so far do not give sufficient statistics, they seem to confirm this order. By collecting more data using the current Chandra and XMM-Newton X-ray observatories, it remains to be seen whether this claim can be sustained.

\subsection{SN 2002ap in NGC 628 (M74)}

At an age of 4 days, the Ic 23] SN 2002ap was detected both in the UV and in X-rays with the Optical Monitor (wavelength range 245-320 nm) and EPIC-pn onboard XMM-Newton 57. Preliminary analysis of the EPIC-pn image showed a $3.5 \sigma$ excess at a distance of $4^{\prime \prime}$ from the optical position of SN 2002ap, below the positional error $\left(<10^{\prime \prime}\right)$ at this stage of the data analysis. Located at a distance of $10 \mathrm{Mpc}$ in the host galaxy NGC 628, the recorded EPIC-pn count rate of $(9.0 \pm 2.5) \times 10^{-4} \mathrm{cts} \mathrm{s}^{-1}$ corresponds to a (0.1-10 keV band) luminosity of $L_{\mathrm{x}} \sim 2 \times 10^{37} \mathrm{ergs} \mathrm{s}^{-1}$ for an assumed power-law spectrum with photon index 2 and a Galactic foreground column of $N_{\mathrm{H}}=5 \times 10^{20} \mathrm{~cm}^{-2}$ [57. A similarly low X-ray band luminosity has been inferred for the Ic SN 1994I (see Sec. 3.7). 


\section{What is so special about X-rays?}

By observing electromagnetic radiation from $\mathrm{SNe}$ (and what else is there in astrophysics, other than neutrinos and gravitational radiation?), we can, in principle, obtain information about (i) the nature and mass of the progenitor, (ii) the possible presence of a companion, (iii) the history of mass loss and the speed of the progenitor's wind going back tens of thousands of years, (iv) inhomogeneities in the CSM, (v) the density gradient of the ejecta as they emerge in the early phase of the SNe, (vi) the geometry, physical conditions and composition of the ejected matter, (vii) temperature and density in the region behind the reverse shock, (viii) speed of the reverse shock as a function of time, (ix) the geometry, physical conditions and composition of the CSM, and (x) the speed of the circumstellar shock as a function of time.

A large part of the story comes from optical observations which historically have played a key role in our understanding and knowledge of the various SNe. The classification of the various 'types' is exclusively linked to the optical (see the Chap. by Turatto). A wealth of information comes directly from the observed IR/optical/UV line profiles and fluxes (see the Chap. by Branch, Baron \& Jeffery).

The radio emission, which is believed to arise largely from the region behind the circumstellar shock, tells its own story (see the Chap. by Sramek \& Weiler). This story is by and large complementary to what we learn from the IR/optical/UV observations. In either wavelength range, there is not always a unique interpretation that explains the complicated spectral behavior. It is therefore perhaps not surprising that Filippenko's earlier review 21] is largely phenomenological - the physics is not always clear, though great progress is being made.

The X-ray observations add something special as they preferentially probe the very hot regions $\left(T \sim 10^{6-10} \mathrm{~K}\right)$ and can thus provide information elusive in any other wavelength regime.

Very roughly, we can expect three phases of X-ray emission: there should be a brief burst of high-energy X-rays from Type Ib/c and II SNe in addition to a black-body continuum of $\sim 0.02 \mathrm{keV}$ as a result of the high-temperature flash associated with the break out of the shock through the stellar surface. In the case of Type Ia, only weak X-ray emission is expected from the prompt thermal detonation (or deflagration) of the compact white dwarf. Such brief prompt bursts have not yet been observed due to the lack of X-ray all-sky monitors and the response time of orbiting X-ray observatories (days). Weeks or months later, high-energy X-rays may be detected when the expanding ejecta have become optically thin to X-rays, and/or when the ejecta plow into CSM supplied by the stellar wind in previous phases of mass loss of the progenitor. The circumstellar shock that is formed in this interaction can have a very high temperature $(T \gtrsim$ $\left.10^{9} \mathrm{~K}\right)$. Radio emission is also believed to come from the region behind this shock. The reverse shock is radiative and a dense, cool $\left(T \lesssim 10^{4} \mathrm{~K}\right)$ shell can form behind the reverse shock [11. If the density gradient of the outer part of a core collapse supernova is very large, the density of the reverse shock will be 
high, and X-rays from the reverse shock will be heavily absorbed in this dense cool shell. When this cool region has expanded sufficiently to become transparent to X-rays, X-ray emission from the reverse shock will start to dominate. Radio emission is not expected to come from this region. Yet, it is remarkable how well the radio and the X-ray emission track each other (see Fig. 1). If there are separate shells of CSM as formed in the past (spasmodic bursts of stellar wind from the progenitor), then the X-ray and radio emission will flare up more than once. In the case of SN 1987A the ejecta are just now beginning to plow into the ring of CSM whose presence was earlier detected with HST [7,8.52.

Immler et al. 36 have put a coherent picture together of the various phases of X-ray emission in the case of SN 1993J. They were even able to reconstruct the evolution of the progenitor of SN 1993J, uncovering $\sim 10^{4}$ years in wind history, and found evidence that the progenitor was making a transition from the red to the blue supergiant phase during the late stage in the evolution. This scenario for the evolution of the SN 1993J progenitor revealed interesting similarities with that of SN 1987A, whose progenitor completely entered the blue supergiant phase after significant mass-transfer to a companion (see the Chap. by McCray). The X-ray data of SN 1993J cover many years, demonstrating the scientific potential of long-term X-ray monitoring of SNe as an important diagnostical tool to probe the CSM interaction and the evolution of the progenitor.

In the case of Type IIn SNe (e.g. SN 1998S), it may not be easy to distinguish line emission that comes from the region behind the reverse shock from line emission that comes from clumpy CSM 12]. The clumpy CSM may have been accelerated to $\sim 10^{2}-10^{3} \mathrm{~km} \mathrm{~s}^{-1}$, in which case the X-ray lines should be Doppler-broadened. The Chandra and XMM-Newton observations, however, did not have a high enough photon statistics to address this question. The Chandra observations of SN 1998S, however, did allow for the unprecedented determination of the abundances of various elements such as $\mathrm{O}, \mathrm{Ne}, \mathrm{Mg}, \mathrm{Al}, \mathrm{Si}, \mathrm{S}, \mathrm{Ar}, \mathrm{Ca}$, and Fe [54]. Comparing the observed abundance ratios with those of the models by Thieleman et al. 171, seems to indicate that the mass of the progenitor was $\sim 18 \mathrm{M}_{\odot}$. This is the first time that a mass determination was made using the X-ray data alone.

\section{Acknowledgments}

We are thankful to Dave Pooley for constructing Fig. 1 from archival, published and propriety right data of the authors, and to Roger Chevalier for helpful discussions.

\section{References}

1. I. Aretxaga et al.: MNRAS 309343 (1999)

2. S. Benetti et al.: IAUC 6170 (1995)

3. E. Berger, S.R. Kulkarni, D.A. Frail: GCN Circ. 1237 (2002)

4. R.M. Bionta et al.: Physical Review Letters 581494 (1997) 
5. J.N. Bregman, R.A. Pildis: ApJ 398 L107 (1992)

6. D.N. Burrows, J. Hayes, B.A. Fryxell: ApJ 450830 (1995)

7. D.N. Burrows et al.: ApJ 452680 (1995)

8. D.N. Burrows et al.: ApJ 543 L149 (2000)

9. C.R. Canizares et al.: ApJ 253 L17 (1982)

10. R.A. Chevalier: ApJ 259302 (1982)

11. R.A. Chevalier, C. Fransson: ApJ 420268 (1994)

12. N.N. Chugai: ApJ 414 L101 (1993)

13. N.N. Chugai: Astronomy Reports 41672 (1997)

14. S. Colgate: Sky \& Telescope 74229 (1987)

15. K. Dennerl et al.: A\&A 365 L202 (2001)

16. T. Dotani et al.: Nature 330230 (1987)

17. A.C. Fabian, R. Terlevich: MNRAS 280 L5 (1996)

18. R.A. Fesen, R.H. Becker: ApJ 351437 (1990)

19. R.A. Fesen, D.M. Matonick: ApJ 407110 (1993)

20. A.V. Filippenko, T. Matheson, A.J. Barth: AJ 1082220 (1994)

21. A.V. Filippenko: ARA\&A 35309 (1997)

22. A.V. Filippenko, E.C. Moran: IAUC 6830 (1998)

23. A.V. Filippenko, R. Chornock: IAUC 7825 (2002)

24. D.W. Fox et al.: MNRAS 3191154 (2000)

25. C. Fransson et al.: ApJ 336429 (1989)

26. C. Fransson, P. Lundqvist, R.A. Chevalier: ApJ 461993 (1996)

27. T.J. Galama et al.: Nature 395670 (1998)

28. P. Garnavich et al.: IAUC 6832 (1998)

29. P. Gorenstein, J.P. Hughes, W.H Tucker: ApJ 420 L25 (1994)

30. M. Hashimoto, K. Iwamoto, K. Nomoto: ApJ 414 L105 (1993)

31. G. Hasinger, B. Aschenbach, J. Trümper: A\&A 312 L9 (1996)

32. K. Hirata et al.: Physical Review Letters 581490 (1987)

33. J.C. Houck et al.: ApJ 493431 (1998)

34. S. Immler, W. Pietsch, B. Aschenbach: A\&A 331601 (1998)

35. S. Immler, W. Pietsch, B. Aschenbach: A\&A 336 L1 (1998)

36. S. Immler, B. Aschenbach, Q.D. Wang: ApJ 561 L107 (2001)

37. S. Immler, A.S. Wilson, Y. Terashima: accepted by ApJ (2002)

38. S. Immler, B. Aschenbach: in preparation

39. P. Kaaret: ApJ 560715 (2001)

40. S.R. Kulkarni et al.: Nature 395663 (1998)

41. S. Kumagai et al.: A\&A 197 L7 (1988)

42. S. Kumagai et al.: ApJ 345412 (1989)

43. B. Leibundgut et al.: ApJ 372531 (1991)

44. M. Leising et al.: ApJ 431 L95 (1994)

45. W.H.G. Lewin et al.: IAUC 6445 (1996)

46. W.-D. Li et al.: IAUC 6829 (1998)

47. P. Lundqvist, R.J. Cumming: 'Supernova Progenitor Constraints from Circumstellar Interaction: Type IA'. In: Advances in Stellar Evolution eds. Rood \& Renzini (Cambridge Univ. Press), 293 (1997)

48. K. Nomoto et al.: ApJ 286644 (1984)

49. R. Petre et al.: PASJ 46 L115 (1994)

50. E. Pian et al.: A\&AS 138463 (1999)

51. E. Pian et al.: ApJ 536778 (2000)

52. P. Podsiadlowski: Nature 350654 (1992) 
53. P. Podsiadlowski, J.J.L. Hsu, P.C. Joss, R.R. Ross: Nature 364509 (1992)

54. D. Pooley et al.: accepted by ApJ, astro-ph/0103196 (2002)

55. A. Ray, R. Petre, E.M. Schlegel: AJ 122966 (2001)

56. A.G. Riess et al.: ApJ 56049 (2001)

57. P.M. Rodriguez-Pascual et al.: IAUC $\mathbf{7 8 2 1}$ (2002)

58. M.P. Rupen et al.: AJ 9461 (1987)

59. I. Salamanca et al.: MNRAS 300 L17 (1998)

60. E.M. Schlegel: AJ 1081893 (1994)

61. E.M. Schlegel: Rep. Prog. Phys. 581375 (1995)

62. E.M. Schlegel:, R. Petre, E.J.M. Colbert: ApJ 456187 (1996)

63. E.M. Schlegel et al.: AJ 1182689 (1999)

64. E.M. Schlegel: ApJ 527 L85 (1999)

65. E.M. Schlegel: ApJ 556 L25 (2001)

66. L.A.L. da Silva: Ap\&SS 165255 (1990)

67. J. Sollerman, R.J. Cumming, P. Lundqvist: ApJ 493933 (1998)

68. R. Sunyaev et al.: Nature 330230 (1987)

69. T. Suzuki, K. Nomoto: ApJ 455658 (1995)

70. Y. Tanaka et al.: IAUC 5753 (1993)

71. F.-K. Thieleman, K. Nomoto, M. Hashimoto: ApJ 460468 (1996)

72. S.D. Van Dyk et al.: ApJ 419 L69 (1993)

73. S.D. Van Dyk et al.: IAUC 5979 (1994)

74. S.D. Van Dyk et al.: IAUC 6386 (1996)

75. S.D. Van Dyk et al.: IAUC 7322 (1999)

76. S.D. Van Dyk: private communication (2001)

77. K.W. Weiler et al.: ApJ 301790 (1986)

78. K.W. Weiler et al.: ApJ 364611 (1990)

79. K.W. Weiler et al.: ApJ 380161 (1991)

80. K.W. Weiler et al.: ApJ 398248 (1992)

81. S.E. Woosley, T.A. Weaver: ARA\&A 24205 (1986)

82. H.-U. Zimmermann et al.: Nature 367621 (1994) 